\title{
Pelvic-Floor Rehabilitation, Part 1: Comparison of Two Surface Electrode Placements During Stimulation of the Pelvic-Floor Musculature in Women Who Are Continent Using Bipolar Interferential Currents
}

Background and Purpose. Electrical stimulation of the pelvic jloor is used as an adjzmct in the conserva! ive treatment of urinal)' incontinence. No comensus exists, however, regarding electrode placements for optimal stimulation of the pelvic floor musculature. The purpose of this study was to compare two dijjèrent hipolar electrode placements, one suggested by Laycock and Green (L2) the other by Dumoulin (D2), during electrical stimulation with inter:ferential currents Q/ the pell $\cdot$ ic-floor musculature in continent women, using a Iwogroup crossover design. Subjects. Ten continentfemale volunteers, ranging in agefrom 20 to 39 years $(X=27.3,5 D=5.6)$, were randomly assigned ta one of two study groups. Methods. Bach s/u\{\{Y group received neuromuscular e!ectrical stimulation (NMES) of the pelvic oor musculature using bath electrode placements, the arder of application heing reversed for each group. Force of contraction was measured as pressure (in centimelers of water [ $\left.\mathrm{cm} \mathrm{H}_{2} \mathrm{O}\right\}$ ) exerted on a vaginal pressure probe attached to a manometer. Data were ana!yzed using a two-way, mixed-model analysis of variance. Results. No difference in pressure was observed between the two electrode placements. D[fferences in current amplitude were observed, with the D2 electrode placement requiring Jess current amplitude toproduce a maximum recorded pressure on the manomeler. Subjective assessment by the subjects revealed a preference for the D2 electrode placement (7 of 10 subject...). Conclusion and Discussion. Tbe lower current amplitudes required with the D2 placement ta obtain recordings comparable to those ohtained with the 12 technique suggest a more comjortab!e stimulation of the pelvic oor muscles. Tbe lower current amplitudes required also suggest that greater increases in pressure might he ohtained with the D2 placement by increasing the current amplitude white remaining within the comfort threshold. Tbese results will help to define treatment guide/ines for a planned clinicat study investigating the ejfècts of NMES and exercise in the treatment of urinary stress incontinence in women postpartum. [Dumoulin C, Seahorne DE, Quirion-DeGirardi C, Sullivan SJ. Pelvic-Jloor rehabilitation, part 1: companson of two swface electrode placements dun'ng stimulation Q/ the peluic oor musculature in women who are continent using bipolar inter:ferential currents. Phys Tber. 1995; 75:1067-10 74.\}

Key Words: Bipolar technique, Electrode position, Inteiferential currents, Pelvic oor electrostimulation, vaginal pressure probe.

\author{
Chantale Dumoulin \\ Derek E Seabome \\ Cécile Quirion- \\ DeGirardi \\ S John Sullivan
}


Urinary continence is the capacity to retain urine in the bladder between two voluntary micturitions. ${ }^{1}$ Incontinence is the involuntary Joss of urine, which can be demonstrated and which presents a social and hygienic problem. ${ }^{2}$

Genuine urinary stress incontinence (GSI) results from urethral sphincter incompetence and is defined by the International Continence Society as "the involuntary Joss of urine occurring when, in the absence of a detmsor contraction, intravesical pressure exceeds the maximal urethral pressure." 2 Genuine stress incontinence is the most common form of urinary incontinence, with an estimated $78 \%$ of cases of GSI being related to pregnancy and the birth process. ${ }^{2}$ Persans with this condition experience incontinence of urine when the intraabdominal pressure is raised, for example, during coughing, sneezing, or any form of physical activity that increases intra-abdominal pressure...

Neuromuscular electrical stimulation (NMES) has been shown to be effective in the treatment of GSI. Stimulation via the pudendal nerve, at frequencies of 20 to $50 \mathrm{~Hz}$, improves urethral closure by activating the pelvic-floor musculature. ${ }^{4}$ In addition, NMES can increase conscious awareness of the action of these muscles, thus facilitating the ability to perform a voluntary muscle contraction." Severa\} methods of stimulating the pelvic-floor muscles have been described, including the use of bath low-frequency faradic currents ${ }^{6.7}$ and mediumfrequency interferential currents.K-W The use of medium-frequency interferential currents has been suggested as a means of overcoming the problem of stimulating deep-seated stmctures more effectively, without using invasive methods. The capacitive component (reactance) of tissue resistance has been hypothesized to decrease inversely with the current frequency. ${ }^{11}$ By decreasing the reactance, the overall tissue resistance will diminish, thereby facilitating the stimulation of cleep stmctures. ${ }^{12}$

Regardless of the method used, the localization of the stimulating electrodes is of critical importance in obtaining a maximal contraction. The intensity of an electrically induced muscle contraction is directly relatee! to the number of motor units activated. ${ }_{3}$ The number of motor units activated, in tum, is influencee! by the current amplitude and frequency and the placement of the stimulating electrodes. ${ }^{13}$

In 1988, Laycock and Green ${ }^{14}$ compared different electrode placements during stimulation with interferential currents of the pelvic-floor muscles of female subjects. Using vaginally located sensors, they measured peak currents and peak pressures evoked in the perivaginal tissues, as weil as tissue resistance, for each of three elec-

C Dumoulin, MSc, PT, b Physical Therapist, Hôpital Ste-Justine de Montrée11, 3175 Côte SteCatherine, Montréal. Québec, Canada H3T !CS, and Teaching Assistant and Lecturer, L'Ecole de Réadaptation, Faculté de Médecine, Université de Montréal, Montréal, Québec, Ce1nada H3C 3]7. Address al! correspondence to Ms Dumoulin at the second address.

DE Seaborne. MSc, PT, is Professor, Depe1rtment of Physiotherapy, L'Ecole de Réadaptation, Faculté de Médecine, Université de Montréal.

C Quirion-DeGirardi, MA, PT. is Associate Professor (ret), L'Ecole de Réadaptation, Faculté de Médecine. Université de Montréal.

SJ Sullivan. PhD, is Associate Professor and Chair, Department of Exercise Science, Concordia University, Montréal, Québec, Canada H4B 1R6, and is affiliated with the Centre de Recherche, Institut de Réadaptation de Montréal, 6300 Darlington Ave, Montréal. Québec, Canada H3S 2]4, and L'Ecole de Réadaptation, Faculté de Médecine, Université de Montréal.

This study was approved by the Ethics Committee of Faculté de Médecine, Université de Montréal.

This article zms submitted Octoher 5. 1994, and was accepted Allf!.USI 15, 1995

trode placements during stimulation of the pelvic floor. They concluded that a bipolar electrode placement, with one electrode placed between the ischial tuberosities (over the anus) and the other electrode placee! over the antenor perineum, inferior to the pubic symphysis, produced an equally effective stimulation of the pelvic floor, as comparee! with a quadripolar electrode placement. They recommended the bipolar placement, basee! on its ease of application. ${ }^{14}$

Electrical stimulation at current intensities necessary to produce adequate muscle contractions can result in unpleasant or painful sensations. Because patient discomfort is otl:en the limiting factor during NMES, 1 " this discomfort can reduce the effectiveness of the treatments. In our clinical practice, we have utilized the bipolar technique suggested by Laycock and Green ${ }^{14}$ for treating women with postpartum GSI. During stimulation, sorne of our patients have complained of intense discomfort due to high current concentration under the anterior electrode (in the region of the clitoris). We have therefore suggestecl an alternative electrode placement for the anterior electrode, to a position immediately superior to the pubic symphysis. We postulate thar this modifiee! electrode placement will decrease the discomfort and increase the efficacy of NMES of the pelvic floor.

The suggested alternative position produces a current spread estimated from anatomical measures to be slightly greater than the $140 \mathrm{~cm}$ (approximate) reported by Laycock and Green. ${ }^{14}$ The direction of current flow follows closely that of the bipolar electrode placement suggested by Laycock and Green. ${ }^{14}$ By displacing the anterior electrode to a point superior to the pubic symphysis, however, the current will theoretically penetrate deeper within the pelvis. ${ }^{11}$ To avoid confusion between these two techniques, for the purpose of this study only, we have reclassified the bipolar female electrode placement suggested 


\begin{tabular}{|c|c|c|c|c|}
\hline Subject No. & Age $\{y)$ & Height (m) & Weight (kg) & BMia $\left(\mathbf{k g} / \mathbf{m}^{2}\right.$, \\
\hline & 24 & $17 n$ & 658 & $23 n$ \\
\hline 2 & 39 & 1.62 & 60.0 & 23.0 \\
\hline 3 & 25 & 1.68 & 61.2 & 21.5 \\
\hline 4 & 25 & 1.57 & 52.1 & 21.0 \\
\hline 5 & 24 & 1.68 & 53.5 & 19.0 \\
\hline 6 & 26 & 1.68 & 56.7 & 20.0 \\
\hline 7 & 20 & 1.60 & 51.3 & 20.5 \\
\hline 8 & 37 & 1.63 & 56.7 & 21.5 \\
\hline 9 & 26 & 1.52 & 49.9 & 21.5 \\
\hline 10 & 27 & 1.70 & 72.6 & 25.0 \\
\hline $\mathrm{X}$ & 27.3 & 1.64 & 58.0 & 21.6 \\
\hline SD & 5.6 & 0.06 & 7.1 & 1.7 \\
\hline
\end{tabular}

"BMI=body mass index.

hy Laycock and Green ${ }^{14}$ as L2 and the alternative electrode placement as 02 .

The purpose of our stuùy was to compare the two different electrode placements (L2 and 02) in the stimulation of the pelvic-floor musculature, using hipolar interferential currents, to determine which of the two methods produœd a stronger contraction with the Iowest current amplitude. The force of contraction of the pelvic-floor musculature was measured indirectly as pressure (in centimeters of water [ $\left.\mathrm{Cm} \mathrm{H}_{2} \mathrm{O}\right]$ ) registered on a manometer attached to a vaginal pressure probe. We expected that 02 would be the more effective of the two electrode placements. The results ohtained from this study helped to determine trealment guidelines for a clinical study of the effects of noninvasive electrical stimulation of the pelvic-floor musculature in women with postpartum urinary stress incontinence (see our companion article in this issue).

\section{Method}

\section{Subjects}

Ten continent women aged between 20 and 39 years $(X=27.3,50=5.6)$, who were recruited from a population of clinicians and graduate and undergraduate university students, volunteered as subjects for this study. Ail subjects demonstrated the ability to perform a voluntary pelvic-floor contraction. None of the subjects had any previous history of urinary incontinence or any neuromuscular injury likely to influence our results. Ail subjects were nulliparous, and during the period of data acquisition, none were menstruating or had an intrauterine deviee implanted. Descriptive statistics are shawn in Table 1. Before participating in the study, ali volunteers signed an approved informed consent form.

Age, weight (wt), and height (ht) were recorded and body mass index (BMI) was computed for each subject. Body mass index ${ }^{16}$ is a measure of obesity

•Enraf-Nonius Delft, f:quipement de Physiothérapie P Gélinas Ltée CP68, Suce "D," Montréal Québec, Canada H3K 3R9.

tMed-0-Gen !ne, H1l MetropolitainE, Montréal, Québec, Canada HIR 1 Z7.

Ponex Ltd, Hythe, Kent, England CT21 6jL

Physical Therapy 1 Volume 75, Number 121 Oecember 1995

and is derived from the formula: wt $(\mathrm{kg}) / \mathrm{ht}\left(\mathrm{m}^{2}\right)$. I3ecause fat hasan electrical impedance of hetween 1,000 and $3,000 \mathrm{O} ! \mathrm{cm}^{2}, 1$ - obesity could have influenced our findings. Ail our subjects had a BMI below 27. Persons having a BMI greater than 27 are consideree! dinically obese. ${ }^{{ }{ }_{\mathrm{i}}}$

\section{Instrumentation}

The electrical stimulator used during this study was an Endomed 433 medium-frequency interferential current stimulator* with a mediumfrequency output of either 2 or $4 \mathrm{kHz}$. According to the manufacturer's specifications, this stimulator has an amplitude modulation frequency spectrum (interference frequency) continuously adjustable between 0 and $100 \mathrm{~Hz}$. A bipolar application implies thar the two medium frequencies are superposed within the stimulator and applied directly as an interferential cmrent at the preselected frequency. The force of contraction of the pelvic-floor musculature elicited by the stimulation was measured indirectly as pressure On centimeters of water [ $\left.\mathrm{cm} \mathrm{H}_{2} 0\right]$ ) registerecl on a manornetert attachee! to a vaginal pressure probe. ${ }^{*}$ The pressure-sensitive manometer used in this study was capable of detecting and measuring changes in perivaginal pressure resulting from contractions of the pelvic-floor muscles. Prior to the experiment, the manometer was examinee! and tested by the hioengineering department of a major Montréal teaching hospital (Hôpital Ste-Justine de Montréal). which reported a high leve! of reliahility for this instrument. Both the manometer and vaginal probe are illustrated in Figure 1.

\section{Experimental Design}

Our experimental design was a twogroup crossover design, with ali subjects receiving stimulation with the two different electrode placements. To reduce any experimental effect resulting from the orcier of stimulation, the 10 subjects were randomly assignee! to one of two groups ( $\mathrm{n}=\mathrm{S}$ per group) prior to the experiment. Each subject selected 1 of a series of 10 sealed envelopes containing an equal num- 


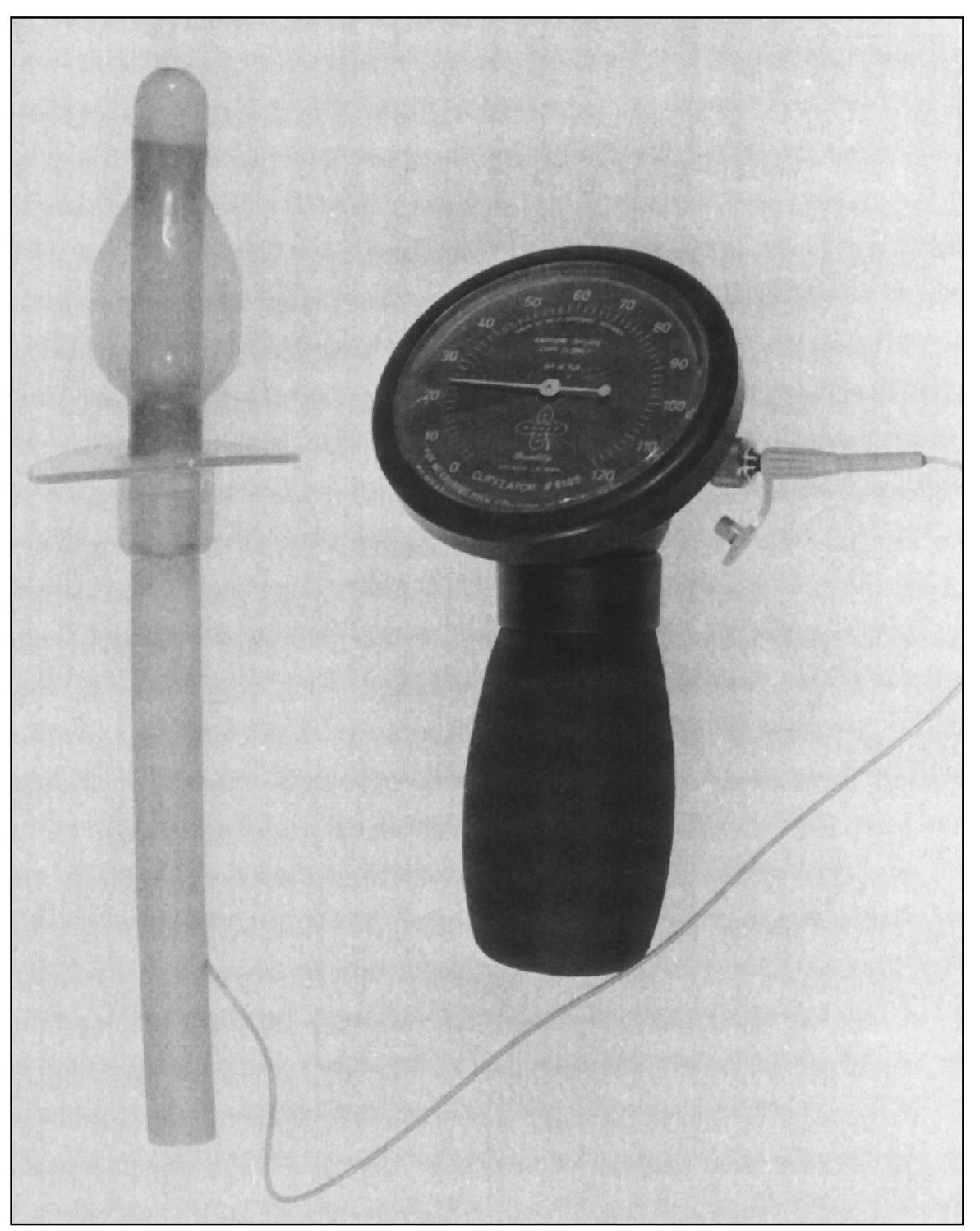

Figure 1. Pressure-sensitive manometer attached to the uaginal pressure probe. ber of odd and even numbers. The 5 subjects who selected an envelope containing an even number began the experiment with the L2 electrode placement, followed by the 02 electrode placement. The 5 subjects selecting an envelope with an odd number were treated in the reverse arder.

\section{Procedure}

Detailed explanations were given to each subject regarding the aims of the study, the equipment to be used, and the techniques. The subject was al- lowed to test the effects of the stimulating current on an exposed portion of her forearm, using a remote current amplitude control. It has been our experience thar patients will tolerate higher current amplitudes if the current is self-regulated. Following this initial briefing, the subject was required to perform her perineal toiler using soap and water, in an adjoining private washroom. She was then instructed to disrobe the lower part of her body and assume a semisupine position (tnmk inclined at $50^{\circ}$ from the horizontal) on a padded wooden
!MUKO Luhricating ]elly, Ingram \& Bell Medical, Don Mills, Ontario, Canada M3B 1L9. treatment table, with the knees and hips supported in flexion at approximately 70 degrees and bath hips in lateral (external) rotation. ${ }^{14}$ This position facilitated the positioning of the electrodes, encouraged relaxation, and reducecl intra-abdominal pressure, $\mathrm{Hl}$ which coule! register on the manometer and thereby influence the results of the study.

Pelvic-floor assessment. The pelvicfloor assessment was performecl by a physical therapist (CD), using a vaginal examination technique clescribed by Chiarelli and O'Keefe. ${ }^{18}$ The therapist wore disposable, sterile surgical latex gloves. After palpating the medial fibers of the subject's pubococcygeus muscle with the index finger, she instructed the subject to contract her pelvic-floor musculature to more accurately identify its precise location.

Accorcling to Bo et al, ${ }^{19}$ the center of pelvic-floor activity is located in an area approximately $3.5 \mathrm{~cm}$ from the introitus.

The disposable vaginal pressure probe was acljusted corresponding to the depth of each subject's musculature, as determinee! by vaginal palpation. Following instructions given by the therapist, the subject then inserted the probe herself, using a sterile watersoluble jelly as a lubricating medium. $\S$ The probe was then attachee! to a manometer. The subject was required to squeeze on the probe by contracting her pelvic-floor muscles. At the same time, the therapist completed the adjustment of the probe position to a site where maximum pressure was obtained, as indicated by the manometer.

\section{Electrode placement and stimula- tion sequence. The electrodes were} placed in position in the predeterminee! arder. The posterior $(6 \times 8-\mathrm{cm})$ carbon-silicone electrode, $*$ enclosed in a cellulose sponge pad* moistened with warm tap water, was placee! directly over the subject's anal region. The anterior electrode $(4 \times 6 \mathrm{~cm})$, similarly enclosecl, was placee! in the median plane, immediately inferior to the pubic symphysis for the L2 technique, or immediately superior to the 


\begin{tabular}{|c|c|c|c|}
\hline \multirow[b]{2}{*}{ Order } & \multirow[b]{2}{*}{ Subject No. } & \multicolumn{2}{|c|}{ Maximum Pressure $\left(\mathrm{cm} \mathrm{H}_{2} \mathrm{O}\right)$} \\
\hline & & L2 & 02 \\
\hline & 2 & 2.5 & 10.0 \\
\hline & 4 & 2.0 & 3.0 \\
\hline \multirow[t]{7}{*}{ L2/D2 } & 6 & 2.0 & 3.0 \\
\hline & 8 & 3.0 & 5.0 \\
\hline & 10 & 40 & 4.0 \\
\hline & $\mathrm{X}$ & 2.7 & 5.0 \\
\hline & $\mathrm{SD}$ & 0.8 & 2.9 \\
\hline & 1 & 4.5 & 5.0 \\
\hline & 3 & 20 & 3.0 \\
\hline \multirow[t]{7}{*}{ D2/L2 } & 5 & 16.0 & 12.0 \\
\hline & 7 & 4.5 & 4.0 \\
\hline & 9 & 5.0 & 6.0 \\
\hline & $\mathrm{X}$ & 6.4 & 6.0 \\
\hline & SD & 5.5 & 3.5 \\
\hline & Grand X & 4.6 & 5.5 \\
\hline & Grand SD & 4.2 & 3.1 \\
\hline
\end{tabular}

"Suh1 ects with even numhers were treated with electrode placement suggcsted by Laycock ancl Green (L2) followecl hy electrode placement suggested by Dumoulin (J)2); subjects with odd numbers weœ tested in the reverse order.

puhic symphysis for the D2 technique. The electrodes were secured in position by rne ms of a perforated ruhber band! passing between the legs of the subject $<$ nd attachee! anteriorly and posteriorly to insulated metal rings on a lumbar traction belt* securecl around the suhject's waist. To avoid any possibility of cross infection between subjects, we used only new cellulose electrode envelopes. which were changed for each subject. The carbonsilicone electrodes and leads were cleaned with alcohol and the pedorated mhber band was disinfected in Cidexll solution following each application.

An amplitude-modulated mediumfrequem:y (AMF) cu1-rent of $10 \mathrm{~Hz}$ and a base frequcncy (carrier frequency) of $2 \mathrm{kHz}$ were used throughout the stucly as the stimulating current. To achieve this AMF cutTent, two separate medium-frequency cwrents, one of $2,000 \mathrm{~Hz}$ and the other of $2,010 \mathrm{~Hz}$, are superposee! within the stimulator. The result is an AMF current rising and falling in amplitude 10 times per second. The effect on the tissues is that of a low-frequency stimulating current of $10 \mathrm{~Hz}$. When muscle tissue is stimulated via the nerve at this frequency, the result is a subtetanic muscle contraction.

Three muscle contractions were elicited with each electrode placement. U.-;ing the remote control, the suhject, under the supervision of the therapist, increased the current amplitude gradually to a leve! of maximum tolerance, remaining at this leve! for 3 seconds. Maximum tolerance was defined as a point just below the pain threshold. To reduce the possibility of any IXTedensky inhibition, 30 seconds only was allowed for each subject to reach

IIJohmon \& Johnson Medical Products, Peterborough, Ontario, Can<tda K9J 7B9.

"SYSTAT !ne, !HOO Sherman Ave, Evanston, IL 60201.

maximum intensity ${ }^{20}$ Wedensky inhibition is the state of incomplete repolarization of a nerve fiher when the nerve is stimulatec.l with a highintcnsity, mcdium-frequency (2,000$\mathrm{Hz}$ ) current. Complete repolarization can only occur if the current intensity is reduccd periodically. ${ }^{21}$ To limit muscle fatigue, a 2-minute rest period separated stimulatecl contractions and a 1)-minute rest pl:'riod separated the two dectrode placement techniques. During this 15-minute period, the electrodes werc removed and the pacls were moistened prior to being secured in the alternative position. At the same time, the vaginal probe was checked to ensure that its position remained unchanged. Of the three contractions elicited in each electrode placement, the strongest contraction was retained for use in the statistical analysis. All readings were verified hy two rescarchers, neither of whom was masked.

\section{Data Analysis}

Descriptive statistics were calculated for maximum pressure and current amplitude. In addition. hoth maximum pressure and current amplitude were analyzed using a two-way, mixedmodel (one hetween-group factor and one within-subject factor) analysis of variance (ANOVA), ${ }^{22}$ the betwcengroup factor heing the orùer of stimulation (L2 folluwed by D2 or D2 followed by L2) and the within-subject factor being the electrode placement (L2 and 02). The ANOVA procedures were perfonned using the SYSTAT statistical package:" A probability leve! of :S:.05 was adoptee! for ali statistical tests.

\section{Results}

Descriptive statistics for maximum pressure and current amplitude are presented in Tables 2 and 3. No difference was ohserved betwcen the maximum pressures ohtained for both the L2 and D2 electrode placements $(P=.34)$, nor was there any effect due to either the orcier of application or the interaction bctwecn the order and the electrode placement (Tah. 4). 
muscular Electrical Stimulation"

\begin{tabular}{|c|c|c|c|}
\hline \multirow[b]{2}{*}{ Order } & \multirow[b]{2}{*}{ Subject No. } & \multicolumn{2}{|c|}{ Current Amplitude (mA) } \\
\hline & & L2 & 02 \\
\hline & 2 & 68 & 45 \\
\hline & 4 & 39 & 39 \\
\hline \multirow[t]{7}{*}{ L2/D2 } & 6 & 60 & 32 \\
\hline & 8 & 53 & 40 \\
\hline & 10 & 61 & 53 \\
\hline & $\mathrm{X}$ & 56.2 & 41.8 \\
\hline & SD & 11.0 & 7.8 \\
\hline & & 50 & 26 \\
\hline & 3 & 16 & 18 \\
\hline \multirow[t]{7}{*}{ D2/L2 } & 5 & 118 & 78 \\
\hline & 7 & 100 & 57 \\
\hline & 9 & 82 & 28 \\
\hline & $\mathrm{X}$ & 73.2 & 41.4 \\
\hline & SD & 40.7 & 25.2 \\
\hline & Grand X & 64.7 & 41.6 \\
\hline & Grand SD & 29.5 & 17.6 \\
\hline
\end{tabular}

"Suhject with en n numbers werc treJtecJ with electrode placement suggeslccl by Laycock and Green (L21 followed lw eleurode placement suggestcd hy Dumoulin (D2); subjects with oclcJ

Table 4. Ana(rsis-C?f-Variance Summaryfor Maximum Prt $>$ ssun! OIJtailled WitiJ Neuromuscular Hlectrical Stimulation

\begin{tabular}{|c|c|c|c|c|c|}
\hline Source & $d f$ & SS & MS & $F$ & $p$ \\
\hline Order & & 27.61 & 27.61 & 1.22 & .30 \\
\hline Error & 8 & 180.75 & 22.60 & & \\
\hline Placement & & 4.51 & 4.51 & 1.35 & .28 \\
\hline Order x placement & 1 & 9.11 & 9.11 & 2.73 & .14 \\
\hline
\end{tabular}

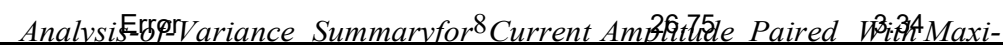

mum Preswre Ohtained With, l'euromuscular Electrical Stimulation

\begin{tabular}{lcrrrr} 
Source & $\boldsymbol{d f}$ & \multicolumn{1}{c}{ SS } & \multicolumn{1}{c}{ MS } & $\boldsymbol{F}$ & $\boldsymbol{p}$ \\
\hline Order & 1 & 344.45 & 344.45 & 0.32 & .59 \\
Error & 8 & 8686.60 & 1085.83 & & \\
Placement & & 2668.05 & 2668.05 & 17.81 & .003 \\
Orderx placement & & 378.45 & 378.45 & 2.53 & .151 \\
Error & 8 & 1199.00 & 149.88 & & \\
& & & & & \\
\hline
\end{tabular}

In contrast, the mean CUITent amplitude required to produce the maximum recorded pressure was found to be less $(. \mathrm{F}=17.81, P<.003)$ with the $\mathrm{D} 2$ placement $(41.6 \mathrm{~mA})$ than that required with the L2 placement (64.7 $\mathrm{rnA}$ ). No elfects duc to the order of presentation or the interaction between order and placement were observed (Tab. 5). These results suggest that the D2 placement was capable of producing a contraction of comparable magnitude at a reduced CUITent amplitude. At the end of the session, when asked which technique they prefcrred, 7 of the 10 women indicated a preference for the D2 electrode placement.

\section{Discussion}

The objective of this study was to determine the most effective of two electrode placements in stimulating the pelvic-floor musculature in continent, nulliparous womcn. The results showed that bath electrode placements achieved contractions of comparable force, as measured by the

manometer. The current amplitude required to achieve the contractions, however, was lower with the D2 electrode placement than with the L2 electrode placement. Based on these results, we concluded that D2 was the more effective electrode placement for these subjects. The subjects' preference for this electrode placement, which they expressed verhally, might also indicate that the D2 electrode placement is also the more acceptable of the two electrode placements, an important consideration in the treatment of female urinary incontinence.

A major problem encountered in attempting to stimulate deep-seated structures, using noninvasive techniques, is the elecrrical impedance olfered by the intervening tissues, which resist the flow of the stimulating current. ${ }^{14}$ The depth and consistency of the tissues between the stimulating electrodes and the motor nerve of a muscle will affect this impedance, thereby influencing the density of current at the target site and thus the quality of the muscle contraction. The motor nerve of the pubococcygeus 




Figure 2. Diagrammatic representation qf'a median sagittal section through the \}i'male pelvis. Arrows indicate the anterior (D2, L2) and posterior (anal) electrode placements. fAdapted.fi-cmzjohnston TB, Whillisj, eds. Gray's Anatomy: Descriptive and Applied. 31st ed. New York, NY·Longmans, Green \& Co; 1956.)

muscle (pudendal) is deeply situated ar a depth of between 7.5 and $10 \mathrm{~cm}$ in the pelvic cavity. 6 Low-frequenc..y srimulating currents (0-50 pulses per second), using a noninvasive electrode placement technique, are incapable of adequately stimulating the pelvic-floor muscles, unless current amplitudes are increased to levels rhat can be extremely painful and porentially harmful to the intervening tissues $\mathrm{f}>$

The problem of tissue resistance has, seemingly, been overcome with the use of medium-frequency interferential currents. 12 The aim of obtaining a maximal contraction of the pelvic-floor musculature with minimal current amplitudes is, however, still desirable, and electrode placement can be of critical importance in achieving this aim.

By displacing the anterior electrode from the anterior perineum, interior to the pubic symphysis (L2), to the region immediately superior to the puhic symphysis (D2), the depth of the current field could, theorctically, he increased. 11.12 Figure 2 illustrates this point diagrammatically. Assuming a gretter depth of penetration, a more effective stimulation of the motor ne1ve to the pubococcygeus muscle might be achieved. A!though no experimental evidence exists to support this hypothesis, a change in direction of the electrical field, produced by displacing the anterior electr $<$ XIe, could explain our results.

Our expectation thar the D2 electrode placement would elicit a stronger muscle contraction than the L2 electrode placement was not realized during this study. No differences were observed between the force of contrdction (measured as pressure in centimeters of water) provoked by the tv..'O electr $<$ XIe placements, nor was rhere any carryover effect from D2 to L2. or vice versa (Tab. 2).

Two possible explanations might account for the lack of any difference in the force of contraction. The instmctions given ro the subjects regarding the effects of the stimulation could have been misundersrood. Follow-up calls to ali subject $<>$, made in an attempt to clarify this point, revealed thar ali subjects ceased increasing the current amplitude upon perception of an appreciable muscle contraction and not necessarily for a maximum contraction. Thus, it is possible thar for the D2 electrode placement, if the current amplitude had been increased to the same levels thar were achieved with the L2 electrode placement, a more forceful contraction would have resulted.

An alternative, or additional, explanation for the submaximal responses is that, when the subjects sensed the contraction, nervousness or the unusual sensation in a very sensitive and intimate region of the body could have caused them to stop increasing the current amplitude, while still weil helow the actual pain threshold, in both electrode placements. Delitto et al"" have shown thar the physical sensation of a muscle contracting as a result of electrical stimulation, comhined with the effect of stimulation of local nociceptors, can lead to apprehension and fear. therehy reducing the effectiveness of NMES as a means of eliciting a maximum or near-maximum contraction. Emotional factors, either consciously or subconsciously, may have influenced our subjects, affecting their comprehension of the instructions or causing them to overreact to the stimulus. In a normal treatment situation, this apprehension and misconception regarding the perceived effects could gradually be overcome with repeated sessions. In such a situation, and with encouragement and guidance, we fee! thar the D2 electrode placement would have resulted in a stronger muscle contraction at current amplitudes still below those obtained with the L2 electrode placement.

The choice of a nontetanizing frequency $(10 \mathrm{~Hz})$ for this study was based on our concem with regard to fatiguing the muscle. Stimulation at higher frequencies $(30-50 \mathrm{~Hz})$ may have resulted in a tetanie muscle contraction, further enhancing the efficacy of the stimulation. However, as any frequenc.y changes would have applied equally to both the L2 and D2 electrode placements, our results would have remained the same.

Dwyer and co-workers 2 have demonstrated thar there is a strong correlation between a high BMI (ohesity) md 
urinal)' stress incontinence in women. Adipose tissue offers a high resistance to current flow,!" and adipose tissue tends to accumulate in the lower abdominal and suprapubic regions in women. ${ }^{2}{ }_{3}$ None of our subjects were classified as obese, but this is a factor that could have influenced our results. Further research should take this aspect into consideration. Another limitation of this study was the restricted number of subjects. Recruiting subjccts for this type of rescarch is difficult, particularly when time constraints are imposed. Our study would have been strengthened had our groupings been larger. In spite of these drawbacks, however, we fee! that the results are encouraging and justify continued evaluation of the D2 technique in clinical trials of interferential currents for the treatment of female urinal)' stress incontinence.

\section{Conclusion}

Two electrode placements for NMES of pelvic-floor muscles have been described and compared, using continent female volunteers as subjects. Equivalent maximum pressures were observed with both electrode placements. Current amplitudes required to obtain maximum pressure readings were Jess using the D2 electrode placement. Our interpretation of these findings is that the D2 electrode placement produces a deeper, and therefore a more precise and effective, stimulation of the pelvic-floor musculature. This interpretation suggesto.; that a stronger muscle contraction might be obtained with the D2 electrode placement in subjects who become progressively more familiar with the stimulation process while undergoing a treatment program. This hypothesis is examined in our companion article in this issue.

\section{Acknowledgments}

We express our appreciation to $\mathrm{Dr}$ Robert Gauthier, Department of Obstetrics, and Dr Yves Homsy, Director, Department of Urology, Hôpital SteJustine de Montréal, for their hclp in the selection and urologie evaluation of the patients participating in this research. Wc also thank Dr Jo Laycock, Bradford Royal Infinnal)', Bradford, England, for her helpful comments and support in the preparation of this article.

\section{References}

1 Hilton P. Unstable urethral pressure: towards a more relevant definition. In: Proceedings of the international Continence Society, Boston Mass. 19H9:37 39.

2 Ahrams P, Blaivas J, Stanton S, Andersen J. The standardisation of the terminology of lower urinary tract function. Scand] Urol Nephrol. 19H8;114:17.

3 Beek RP, Hsu N. Pregnancy, childbirth, and menopause related to the development of stress incontinence. Am] Ohstet Gynecol. 1965;91:820 823.

4 Fall M, Lindstrom S. Electrical stimulation. Urol Clin N011h Am. 1991;18:393 407.

5 Leriche $\mathrm{A}$, Leriche $\mathrm{B}$. Place à la rééducation dans l'incontinence urinaire de la femme. J Uro/ (Paris) 1988;94:285 288.

6 Scott B, Green V, Couldrey B. Pelvic faradism: investigation of methods. Physiotherapy. 1969;55:302 305.

7 Plevnik S, Janez J, Vrtacnik P. Sh011-term electrical stimulation: home treatment for urinary incontinence. Worldj Urol. 1986;4:24 26. 8 McQuire W. Electrothera py and exercises for stress incontinence. Ph siothera py. 1975: 61:305 307.

9 Laycock J. Assessment and Treatment of Pelvic Floor Dy;function. Bradford, England:
Postgraduate School of Biomedical Sciences, Bradford University; 1992. Doctoral thesis. 10 Olah K, Bridges N, Denning J, et al. The conservative management of patients with symptoms of suess incontinence: a randomized, prospective study comparing weighted vaginal cones and interferenti<II therapy. Arnj Ohstet Gynecol. 1990;162:87 92.

11 Meyer-Waarden K, Hansjurgens A, Friedmann B. Representation of electric fields in inhomogenous biological media. Biomed Tech (Berlin). 1980;25:295 297.

12 Goats GC. Interferential current therapy. lJr] Sports Med. 1990;2:87 92.

13 Barnetr S, Cooney K, Johnston R. Elecrrically elicited quadriceps femoris muscle torque as a function of various electrode placements . .f Clin Hlectrophysiol. 1991;3:5 8.

14 Laycock J, Green R. Interferential rherapy in the treatment of incontinence. Phvsiotherapy. 1988;74:161 168.

15 Delitto A, Strube M, Shulman A, Minor S. A study of discomfort with electrical stimulation. Phys 1ber. 1992;72:410 424.

16 Health and Welfare Canada. Weight Levels Associated With Health: Canadian Guide/ines 1988. Ottawa, Ontario, Canada: Supply and Services Canada; 19i19:26. Catalogue no. H3'J 134.

17 Baker LL. Neuromuscular electrical stimulation in the restoration of purposeful limb movement. ln: Wolf SL, ed. Hectrotherapy. New York, NY: Churchill Livingstone !ne; 1981:2548

18 Chiarelli P, O'Keefe D. Physiotherapy for the pelvic floor. Australian Journal of Physiotherapy. 1981;27:103 108.

19 Bo K, Hagen R, Jirgensen .J, et al. The effects of two different pelvic floor muscle exercise programs in the treatment of urinary stress incontinence in women. Neurology and Urodynamics. 1989;H:355 5S6.

20 Kloth L. Interference current. ln: Nelson RM, Currier DP, eds. Clinicat Hlectrotberapv. East Norwalk, Conn: Appleton \& Lange; 1987: 183207.

21 Hansjurgens A, May liU. 'li-aditional and Modem Aspects oj'Electrotherapy 2nd ed. Temecula, Calif: Nemectron Medical !ne; 1984: 37

22 Myers J. Fundamentals oj'fuperimental Design. 2nd ccl. Boston, Mass: Allyn and Bacon !ne; 1973:1'!1 196.

23 Dwyer P, Lee E, Hay D. Ohesity and urinary incontinence in women. Hrj Ohstet Gynaecol. 1988;95:9196. 